

\title{
Una mirada a la enseñanza de la historia nacional cubana
}

Volumen $6 \mathrm{~N} .{ }^{\circ} 44$ enero - junio de 2018

ISSN: 0122-4328

ISSN-E: 2619-6069

pp. 37-46

\author{
A Look at the \\ Teaching of National \\ Cuban History
}

Una olhada a ensino

da história cubana

Josefa Azel Jiménez*

Ángel Guido Navarro Otero"*

Fecha de recepción: 31-10-2017

Fecha de aprobación: 20-09-2018

\section{PARA CITAR ESTE ARTÍCULO}

Jiménez, J. y Navarro, A. (2018). Una mirada a la enseñanza de la historia nacional cubana. Nodos y Nudos, 5(44), 37-46.
Doctora en Ciencias de la Educación. Departamento de Historia. Universidad Central "Marta Abreu" de Las Villas, Cuba.

josefaa@uclv.edu.cu.

Doctor en Ciencias Pedagógicas. Director Docente Metodológico Universidad Central "Marta Abreu" de 


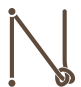

Volumen $6 \mathrm{~N} .{ }^{\circ} 44$ enero - junio de 2018 ISSN: 0122-4328 ISSN-E: 2619-6069 pp. 37-46

\section{RESUMEN}

En este artículo se ofrece un panorama histórico acerca de la enseñanza de la asignatura Historia de Cuba, desde la segunda mitad del siglo xIx hasta la actualidad. Resalta la necesidad de impartirla por sus potencialidades para transmitir valores como una preocupación permanente del magisterio cubano desde que Cuba estaba sometida al yugo colonial español. En los inicios de la República, la enseñanza de la historia nacional y local no fue una estrategia educacional en los gobiernos de turno, pero gracias a la labor de Ramiro Guerra fue incluida en los planes de estudio de la enseñanza primaria. Con el triunfo de la Revolución Cubana en 1959, se redimensionó la asignatura; hoy, se encuentra insertada en los modelos curriculares de los distintos niveles de enseñanza.

Palabras clave: enseñanza; historia; valores

\section{ABSTRACT}

This paper offers a historical overview of the teaching of the subject History of Cuba from the second half of the nineteenth century to present times. It highlights the need to impart it for its potential to transmit values as a permanent concern of Cuban teachers ever since Cuba was subjected to the Spanish colonial oppression. In the early days of the Republic, the teaching of national and local history was not an educational strategy of the successive governments but, thanks to the work of Ramiro Guerra, it was included in the primary education curricula. With the triumph of the Cuban Revolution in 1959, the subject was redesigned; nowadays, it is inserted in the curriculum models of the different levels of education.

Keywords: teaching; history; values

\section{RESUMO}

Este artigo oferece uma visão histórica do ensino da disciplina História de Cuba a partir da segunda metade do século XIX até o presente. Destacamos a necessidade de ministrá-la pelo seu potencial de transmitir valores como uma preocupação permanente do ensino em Cuba desde que o país foi submetido ao jugo colonial espanhol. Nos inícios da República, o ensino da história nacional e local não era uma estratégia educacional nos governos da época, mas graças ao trabalho de Ramiro Guerra foi incluída nos currículos do ensino primário. Com o triunfo da Revolução Cubana em 1959, a disciplina foi redimensionado; hoje, está inserida nos modelos curriculares dos diferentes níveis de ensino.

Palavras-chave: ensino; história; valores 


\section{Introducción}

En la concepción curricular de diferentes modelos educativos en diversas regiones y países, se incluye la enseñanza de la Historia y especialmente, la historia nacional. De ahí que la asignatura Historia de Cuba, caracterizada por el estudio de la aspiración al ser nacional cubano, cuente con una fuerte presencia en los currículos desde la educación primaria hasta la universitaria, donde se seleccionan con un sentido didáctico los contenidos referidos a la historia nacional y local que permitan la educación histórica de los niños, adolescentes y jóvenes.

Este ha sido el resultado de una ardua tarea que llega a nuestros dias, pues el proceso de enseñanza/ aprendizaje de la asignatura Historia de Cuba apareció en el siglo XIX, pero no siempre se enseñó de la forma que hoy se hace, dista mucho de lo que se presupone en la actualidad. Un recorrido a través del tiempo permitirá comprender su evolución y desarrollo, por lo que en el presente artículo se ofrece un panorama de la enseñanza de la asignatura desde la segunda mitad del siglo xIx hasta la actualidad.

\section{La enseñanza de la asignatura Historia en la etapa colonial}

El proceso de conquista y colonización de Cuba produjo un cambio histórico en la evolución de la pacifica población indigena que habitaba la isla. Se impusieron transformaciones a través de la fuerza brutal, la cultura aborigen fue prácticamente extinguida, algunas de sus costumbres y mitos, así como ciertos relatos de las crónicas de los conquistadores se reflejaron escasamente en los primeros textos escolares de la educación elemental en la etapa colonial. Fue preciso que pasaran más de cuatro siglos para que se diera a conocer la historia de la isla a través de su enseñanza en la escuela.

La asignatura Historia se introdujo en el currículo escolar a finales del siglo XVIII aunque en realidad se estableció como disciplina curricular con el plan de estudio de 1842 y la secularización de la universidad, al implantarse la enseñanza de la Historia de España como asignatura para todo el sistema educacional de la Colonia, con el fin de liquidar las bases del movimiento intelectual ilustrado cubano y las expresiones independentistas de principios de siglo.

En su enseñanza aprendizaje, se realizaba un estudio memorístico de biografias de personalidades destacadas, se enaltecian los sentimientos hacia la metrópoli, se exaltaba a la realeza española y a los grandes conquistadores. Sin embargo, se excluian los pasajes brutales de la conquista y colonización.

Este plan de estudio fue ratificado en las reformas educativas de 1863 y 1880, por lo que a la enseñanza de la Historia con el concepto de hispanidad se le incorporó la de Cuba y Puerto Rico que se impartiría en la primaria elemental y superior. La Historia Universal y la Historia de España quedaban para el bachillerato y la universidad.

La concepción curricular aplicada demostró que la asignatura Historia de Cuba era una historia regional precedente a la Historia de España y a la Historia Universal. Su impartición respondía a la política colonialista, al pensamiento cultural hispánico, a los criterios históricos de los representantes antipatrióticos de la oligarquia criolla vinculada al poder económico y a una metodología basada en la concepción educativa de la escolástica española, por lo que era repetitiva, irreflexiva y memorística.

Los textos escolares transmitían creencias, valores y representaciones de la Historia de Cuba que garantizaban un proceso de asimilación político-cultural y una conciencia histórica hispánica. Consideraban a Cuba como una región de España, cuyo futuro y progreso se basaban en el entendimiento, la cordialidad y la alianza entre criollos y peninsulares para así evitar cualquier inquietud independentista.

A pesar de las imposiciones coloniales, los educadores cubanos comprendieron tempranamente las potencialidades de la historia para transmitir valores, sentimientos y actitudes, sobre todo para la formación del patriotismo y la defensa de la nacionalidad cubana. José Agustín Caballero, Félix Varela Morales, José de la Luz y Caballero fueron los pensadores principales, los iniciadores de los ideales de cubanía de las nuevas generaciones, ya que contribuyeron a preparar las conciencias para los cambios, comprendieron la importancia de la historia en la formación de valores y se valieron de la educación para emprenderlo. 
José Martí, el Apóstol de la Independencia de Cuba, le confirió especial importancia a la enseñanza de la Historia y dejó constancia cuando escribió: "Esta es una humanidad que se desenvuelve y se concentra en estaciones y en fases. Lo de hoy es necesario estudiar en algo los residuos de las sociedades que han vivido" (Martí, 1963, p. 76).

Sin duda, el ideal hispánico escolástico impuesto para la enseñanza de la Historia a través de sus textos y metodología recibió una fuerte oposición en el transcurso del siglo xIx por parte de muchos educadores cubanos, cuyas ideas educativas habian evolucionado desde la llustración y el positivismo crítico, hasta llegar al avanzado pensamiento pedagógico de Martí.

La intervención norteamericana en la guerra de independencia de los cubanos contra el yugo colonial español frustró su inminente victoria, ratificada con el Tratado de París, pues Cuba dejaba de ser colonia española, pero era no libre ni independiente todavía. Se iniciaba así la primera ocupación militar norteamericana que preparó las condiciones para convertirla en una neocolonia, ya que consolidó el predominio económico norteamericano y cercenó su soberanía con la Enmienda Platt.

A su vez, se aprovechó la pésima situación educacional para establecer el sistema de escuelas públicas, se trató de imponer las concepciones y proyectos educativos encaminados a norteamericanizar a los cubanos desde edades tempranas. La enseñanza de la Historia fue el instrumento idóneo para fomentar sus intereses al introducir de forma obligatoria la Historia de Estados Unidos en las escuelas públicas como una de las medidas del gobierno de ocupación. Asimismo, fueron seleccionados algunos maestros primarios para que recibieran cursos de preparación en las Universidades de Harvard y Cambridge, de Estados Unidos que servirian para influir en la educación elemental cubana (Chávez, 2001, p. 83).

Esta etapa fue identificada como "uno de los capítulos más dramáticos y conflictivos de la educación en ese periodo" (Guzmán, 2001, p. 26). Se ofreció una versión manipulada de la "Historia de Cuba" donde se otorgaba relevancia al papel salvador de Estados Unidos en la obtención de la independencia. La enseñanza de la Historia y las personalidades históricas se caracterizaba por el estudio de anales y descripciones sustentados filosóficamente por el positivismo y el pragmatismo.

La Orden Militar No. 226, del 6 de diciembre de 1899, y el Manual para el Maestro, en 1900, constituyeron los documentos rectores para la impartición de la Historia en las escuelas. Contenían las orientaciones didácticas para su aprendizaje, aunque no poseían una adecuada dosificación ni graduación de sus contenidos. Se enseñaba la Historia de Cuba con textos escritos por españoles o norteamericanos basados en sus criterios y versiones; disminuian el estudio de las etapas de luchas independentistas del pueblo cubano, y se impartía la asignatura vinculada e ilustrada con los hechos, procesos y personalidades de Estados Unidos.

En el Manual no se plasmaron orientaciones metodológicas especificas para la enseñanza de la Historia de Cuba. No obstante, expresaba claramente:

Por mucho que se recomiende el cosmopolitismo en la enseñanza de la historia, adviértase que la de la localidad y la de la patria en general han de ser objeto en todos los grados de mayor consideración porque en último resultado, nos ha de interesar más nuestro país que los ajenos. (Guzmán, 2001, p. 28).

\section{La enseñanza de la asignatura Historia en la Cuba republicana}

En 1902, al establecerse la República Neocolonial, se aplicó un nuevo plan de estudio rectorado por la Circular No. 5, dictada por la Junta de Superintendentes de Escuelas de Cuba el 20 de mayo de 1901 para las escuelas públicas primarias elementales. La asignatura Historia de Cuba quedaba excluida del currículo escolar y, en su lugar, se incluía la Historia de América que favorecía los conocimientos sobre la sociedad de Estados Unidos y limitaba la enseñanza de la historia nacional, por lo que constituyó un retroceso en relación con el Manual de 1900.

En los siguientes planes de estudio aprobados en los primeros años republicanos para los diferentes niveles de enseñanza, la historia local no fue casi atendida en las orientaciones metodológicas, ni 
adecuadamente considerada en la instrumentación curricular de los programas diseñados. En la primaria fue ignorada completamente.

Con la aplicación de los cursos de estudios de Historia normados por la Circular No. 76 de 1914, se orientó, de manera muy simple y sin la importancia requerida, abordar en los primeros grados algunos aspectos de la historia local como agregados a la Historia de Cuba. Esta situación empeoró con los nuevos cursos de estudios que para este nivel instituia la Circular No. 105 de 1922, donde se le restó importancia a este tema.

A pesar de esto, desde 1899, una vanguardia del magisterio cubano y especialmente en las escuelas públicas, el maestro enseñó las mejores tradiciones de lucha de su pueblo, enaltecía a los héroes legendarios de la nación y recomenzó a formar las nuevas generaciones que marcarian la marcha en las luchas contra el dominio neocolonial (García, 1980, p. 57).

Según explica el historiador Ramiro Guerra (1950) con la aprobación de la Circular No. 114 de 1926, se modificaron los cursos de estudio de las escuelas primarias públicas rurales y urbanas. La asignatura de Historia de Cuba se iniciaba en tercer grado con la historia local, y en cuarto, quinto y sexto grados, la nacional. Con la inclusión de la historia local, se iniciaba el estudio de personalidades históricas de la comunidad, no solo los que se destacaron por sus acciones políticas, sino también los que realizaron aportes en la esfera social.

La circular restablecía el plan de estudios de 1914 con determinadas modificaciones, ya que el de 1922 presentaba graves insuficiencias. El curso de Historia de Cuba se rediseñó integramente y según asevera Ramiro Guerra en su obra La Educación Primaria en el siglo xx (1950), fue objeto de alteraciones sustanciales al introducirle los estudios de la historia local como punto de partida de la enseñanza y al proporcionarle un nuevo ordenamiento a los contenidos de estudio en los últimos grados.

Ramiro Guerra y otros autores se refirieron a las ventajas del estudio de la historia local, ya que esta despertaría en el niño el interés por la historia nacional, le ayudaría a comprender los hechos del pasado, el valor de la historia; sería fuente de inspiración y patriotismo; además enseñaría a observar, a investigar y a vincularlo con la comunidad.

Este proyecto curricular con algunas variantes estuvo vigente hasta 1958. Sin embargo, la inclusión de la historia local en el tercer grado presentó dos grandes inconvenientes: la falta de preparación de los maestros en esta materia y la ausencia de textos.

Los pedagogos cubanos comprendieron a cabalidad esta problemática, por lo que la Junta de Superintendentes de la República orientó, primero, que los maestros acudieran a las fuentes primarias $y$, segundo, que se recurriera a la historia oral. Además, ninguno de los dos textos básicos de la enseñanza de la Historia de Cuba durante la República, Nociones de Historia de Cuba, de Vidal Morales, e Historia elemental de Cuba, de Ramiro Guerra, llegaron a abordar los contenidos relacionados con las localidades específicas.

Los lineamientos establecidos a partir de 1944 con la Circular No. 133 destacaban la importancia de la enseñanza de la Historia de Cuba, al señalar: "[...] el alumno encontrará un fecundo manantial de instrucción, reflexión y satisfacciones espirituales que contribuirán a despertar en él, el amor a su pueblo y el deseo de contribuir a su engrandecimiento [...] poderoso factor de formación cívica y moral" (Guzmán, 2001, p. 31).

No obstante, esta aseveración pedagógica fue tergiversada muchas veces con enfoques historiográficos conservadores que, además, excluyeron los ideales antianexionistas y antiimperialistas de los próceres de la Independencia y no tuvieron en consideración a hombres destacados de los primeros años republicanos.

En el proyecto investigativo en curso "Reedición crítica de la obra de Ramiro Guerra" se analizaron los aportes de este historiador y pedagogo cubano en este periodo republicano, dirigidos a la escuela primaria, a la organización de planes de estudio y programas, a la investigación, a la redacción de varias obras, así como a la enseñanza aprendizaje de la Historia en este nivel.

Este autor destacaba la contribución de las clases de Historia para la formación de sentimientos morales y patrióticos, pues consideraba que debian estar llenas de vida para que los niños pensaran y sintieran como los grandes hombres, se emocionaran con las hazañas de los héroes que gozaran sus victorias 
y sufrieran sus derrotas. En resumen, que sintieran admiración por la historia de su patria.

En estos años, se destacaron historiadores y pedagogos como Miguel A. Cano, Pedro Garcia, Alfredo M. Aguayo, Emilio Roig, Fernando Ortiz, Raúl Ferrer, Gaspar J. García Galló, entre otros. Muchas de sus contribuciones se refieren al factor emocional en la enseñanza de la Historia y al valor de la oralidad en las clases, aspectos que fueron recomendados en las obras escritas para las Escuelas Normales de Maestros. Se coincide por tanto, con que "en el acto de narrar, de transitar del recuerdo a la palabra [...] con las voces del pasado, de la cultura y de la comunidad" (González, 2016, p. 109), se requiere del sentir del maestro, de su apasionamiento y vehemencia.

En los inicios de la República, la enseñanza de la historia nacional no fue una estrategia educacional en los gobiernos de turno, pues en la primaria superior su presencia fue tardía e irregular, precisamente hacia la década de 1930. Ramiro Guerra, superintendente general de Escuelas, propuso los cursos de estudios para las Escuelas Primarias Superiores que fueron aprobados en 1931. Se indicaba propiciar conocimientos a los alumnos acerca de la evolución de la organización gubernamental y la vida política del municipio, la provincia y la nación cubana.

Los programas trataban de integrar las asignaturas de Historia, Geografía y Cívica en los dos grados de ese nivel donde predominaban los aspectos cívicos y políticos sobre los históricos, aunque muy limitado a determinados recuentos acerca de la evolución de las instituciones municipales y provinciales en Cuba, por lo que no constituyeron una unidad lógica de la historia local iniciada en tercer grado de la escuela primaria.

En relación con la segunda enseñanza o bachillerato, la asignatura Historia de Cuba no estaba contemplada en el plan de estudio diseñado por Enrique José Varona en 1901, su incorporación se efectuó en el curso escolar 1939-1940. Respecto a la educación técnica, se puede afirmar que la asignatura fue ignorada en algunas ramas. En la enseñanza superior, su introducción se produjo en 1927, limitada a determinados estudios o especialidades de la Universidad de La Habana, única existente en el país en esos años.

\section{La Revolución Cubana y la enseñanza de la asignatura Historia}

El triunfo de la Revolución en 1959 originó un viraje trascendental en la educación cubana, se efectuó la colosal campaña de alfabetización en 1961, se institucionalizó y se reorganizó el sistema nacional de educación. En este proceso se redimensionó la enseñanza de la Historia de Cuba y de las personalidades históricas, se transformaron radicalmente las fuentes historiográficas, se le otorgó un papel principal al estudio de las mejores tradiciones de lucha del pueblo cubano y sus líderes principales.

Se llevó a cabo un trabajo encaminado a investigar, recuperar, completar, reactualizar y enseñar aspectos de la historia omitidos o tergiversados, por lo que el Gobierno Revolucionario implementó una serie de medidas para lograr su cometido.

Se realizaron serios esfuerzos para perfeccionar la enseñanza de la Historia en los primeros años de la Revolución en el poder a través de la formación emergente y superación de los docentes de la especialidad, así como en la actualización de los planes y programas de estudio basados en los aportes a la historia nacional efectuados por eminentes estudiosos como Julio Le Riverend, Emilio Roig, Manuel Moreno Fraginals, Juan Pérez de la Riva, Fernando Portuondo, Hortensia Pichardo, Jorge Ibarra y otros.

La asignatura Historia de Cuba estuvo presente en el diseño curricular de la enseñanza primaria y secundaria, en el preuniversitario se impartió por algún tiempo la Historia Económica de Cuba. Se comenzaron a atender sistemáticamente las dimensiones espaciales, temporales y causales de la historia para su estudio y se desarrollaron interesantes experiencias metodológicas, pero también se presentaron algunos inconvenientes.

En relación con la historia local, su enseñanza no se concibió ni se instrumentó en los planes de estudio, pues faltaban condiciones para desarrollar un óptimo proceso de enseñanza/aprendizaje producto de que aún eran insuficientes los estudios relacionados con las historias regionales y locales, lo que implicaba la escasez de temáticas históricas de la localidad 
para abordar la enseñanza; la textolografía escolar de la asignatura Historia no avanzó a la par de la historiografía; las limitaciones académicas que presentaban los docentes; los planes de estudio para la formación profesoral no contemplaban la enseñanza de la historia local ni tampoco su metodología para el proceso de enseñanza aprendizaje.

Con el perfeccionamiento del Sistema de Educación a partir del curso 1976-1977, se produjeron cambios en el diseño curricular para la enseñanza de la asignatura, los conocimientos históricos se enseñaban de primero a cuarto grado a través de efemérides y escritos en los libros de lectura; a partir de quinto grado se comenzaba con Historia Antigua y Media; de sexto a noveno se impartía el resto de las etapas de la Historia Universal que se alternaba en cada grado con las diferentes etapas de la Historia de Cuba, lo que incidió negativamente en la asignatura como tal, ya que perdió su presencia en la enseñanza primaria.

Como puede apreciarse, en esta etapa el estudio de la historia nacional fue minimizado en la educación primaria, así como en la secundaria básica y en el preuniversitario tuvo que integrarse a alternar su espacio con la Historia Universal. Por otra parte, predominó su estudio a partir de la relación entre causas y consecuencias; se introdujo el estudio de grandes personalidades históricas de la Edad Antigua, Media, Moderna y Contemporánea. En los textos correspondientes no se exponian biografías, ya que en el análisis de los hechos se insertaban las actuaciones de los grandes hombres.

Con la riqueza historiográfica legada por prestigiosos historiadores de la primera mitad del siglo xx y la intensificación de los estudios históricos nacionales, pero sobre todo locales en el último cuarto de ese siglo, la ciencia histórica puso a disposición de la escuela una variedad de fuentes para estudiarla y un sinfín de temáticas que desde lo educativo contribuyen a la educación de los niños, los adolescentes y los jóvenes.

Eso contribuyó a que ya en el curso escolar 19881989 se aplicara un nuevo diseño curricular donde la Historia de Cuba recuperó su espacio e independencia en todos los niveles de enseñanza del Sistema Nacional de Educación, se indicaba en sus objetivos y temáticas la atención a la historia local, por lo que el diseño asumido para la enseñanza/aprendizaje de la Historia, particularmente de la historia nacional, fue coherente y ordenado desde la escuela primaria hasta la educación superior, lo que posibilitó abordar los elementos de la historia local a partir de los objetivos formativos.

Los programas de Historia de Cuba utilizados en todas las enseñanzas del Sistema Nacional de Educación se caracterizan por una mayor actualización de los sistemas de conocimientos y habilidades, mejor precisión de los objetivos educativos y novedosas concepciones metodológicas basta citar el modelo didáctico de la enseñanza de la asignatura, cuya finalidad educativa es el desarrollo integral del alumno, el crecimiento de su personalidad, su formación humanista. Además, se orienta para cada unidad de estudio el tratamiento de la historia local sobre la base de la flexible y adecuada selección del sistema de conocimientos por parte de los profesores.

A partir de 1996, la enseñanza de la Historia de Cuba se declaró como una prioridad y una dirección principal del trabajo metodológico para el Sistema Nacional de Educación con vistas a fortalecer la formación integral de los niños y jóvenes, la identidad nacional y preservar las raíces históricas del pueblo cubano a través de un correcto proceso de enseñanza/ aprendizaje de la asignatura, pues "una buena clase de Historia es hija de la libertad y nieta de la cultura, porque esta es la madre de la libertad" (Díaz, 2002, p. 20).

La enseñanza de la Historia debe revelar en cada clase la moralidad histórica del pueblo, de sus héroes, valorar las figuras y los hechos en que participaron para lo cual deben conocer sus acciones, sus ideales y el contexto en que desarrollaron su labor. Relacionar la grandeza del pueblo y los patriotas como seres humanos con sus vivencias y las necesidades de hoy es lo que los hace imitables, alcanzables y reales. La clase de Historia debe dejar una lección: contribuir a elevar la condición humana. La Historia es la gran tradición, de ahi su riqueza; es la memoria de los pueblos.

Lo antes planteado se puede lograr a través de una autopreparación efectiva del docente que le garantice contar con una cultura histórica necesaria para tomar las decisiones didácticas más acertadas 
y dé un aprendizaje reflexivo, colaborativo, de intercambio constante con y entre sus alumnos. En general se aspira al protagonismo fluctuante profesor/ alumno sobre la base de un enfoque comunicativo/ axiológico.

En la primera década del siglo XXI, se estableció la enseñanza de la asignatura Historia de Cuba en la educación superior para todas las carreras universitarias, incluyendo las de ciencias técnicas y agropecuarias, consenso que se llegó al tener en cuenta la necesidad del conocimiento de la sociedad por parte de cualquier profesional, técnico, obrero y campesino independientemente de la actividad laboral que desempeñe en la sociedad.

El proceso de enseñanza/aprendizaje de la Historia de Cuba en las universidades contempla un amplio y coherente programa que abarca desde 1492 hasta la actualidad. Para su dosificación se asumió una correcta utilización del sistema de conocimientos planteados en cada uno de los seis temas en que se divide dicho programa y se distribuyen en las diferentes formas de organización de la docencia universitaria: conferencias, seminarios, clases prácticas, fundamentalmente.

El contenido se inicia con un panorama general de la Cuba colonial de los siglos XVI al XVIII, transitando por las luchas de liberación nacional del pueblo cubano, la República Neocolonial hasta llegar a la Revolución en el poder desde 1959 hasta principios del siglo XXı. Cada tema pretende el estudio de la realidad cubana mediante la comprensión de sus problemáticas esenciales en una relación pasado/presente que le permita al futuro profesional participar creadoramente en la lucha ideológica, cultural y educativa de estos tiempos.

Se aspira a un hombre que responda a su época que defienda la identidad como cubano y latinoamericano frente a las fuerzas disolventes internas y externas que pretenden su desnaturalización cultural y humana, y esto se puede lograr con la enseñanza de la Historia.

A pesar del lugar especial que tiene la Historia en el currículo cubano, los resultados en su aprendizaje no están en el nivel deseado. Por esto, a partir de la política educacional cubana se continúan realizando acciones para elevar el dominio del contenido histórico de los alumnos en los diferentes niveles educacionales.

\section{Conclusiones}

El análisis de la enseñanza de la Historia de Cuba y de las personalidades en la escuela primaria en la etapa colonial revela el papel que ha desempeñado en la formación del patriotismo y de la nacionalidad, especialmente como portadora de valores, sentimientos y actitudes a las nuevas generaciones que se formaban.

En la etapa republicana se diferencian dos posiciones acerca de la enseñanza de la Historia: los defensores de la nacionalidad, seguidores de la tradición pedagógica cubana con el implícito carácter de la educación axiológica y los partidarios conscientes o inconscientes, de asimilar la didáctica norteamericana o europea basada en sus criterios o versiones tergiversadas de la verdadera historia. Se utilizaron, esencialmente, los métodos orales repetitivos, memorísticos y reproductivos.

En las aulas de los mejores maestros cubanos, la enseñanza de la Historia sirvió como un acicate para la defensa de los intereses de la nación cubana. A pesar de que existieron influencias foráneas de dominación, se enfatizó en las emociones y sentimientos, reflexiones, análisis y búsqueda de la autoctonía.

Las transformaciones educacionales ocurridas después del triunfo revolucionario de 1959 modificaron los enfoques historiográficos, pedagógicos, filosóficos y metodológicos acerca de la enseñanza de la Historia y sus personalidades enmarcadas en un proceso paulatino de perfeccionamiento y elevación de la calidad de su enseñanza que llega hasta nuestros días.

\section{Referencias}

Chávez, J. (2001). Bosquejo histórico de las ideas educativas en Cuba. La Habana, Cuba: Editorial Pueblo y Educación.

Díaz, H. (2002). Enseñanza de la historia nacional: un enfoque desde lo local. La Habana: Editor Educación Cubana.

Garcia, G. (1980). Bosquejo histórico de la educación. La Habana: Editorial de Libros para la Educación.

González, M. (enero-julio de 2016). Narrar-nos es formar-nos: las historias de vida en la formación de maestros. Nodos y Nudos, 4(40), 103-116.

Guerra, R. (1923). La defensa nacional y la escuela cubana. La Habana: Libreria Cervantes.

Guerra, R. (1950). La Educación Primaria en el siglo Xx. La Habana: Libreria Cervantes. 
Guerra, R. (1952). Historia de la nación cubana. 10 tomos. La Habana: Editorial Historia de la Nación Cubana.

Guzmán, L. (2001). Historia de Cuba. Temas metodológicos para maestros primarios. La Habana: Editorial Pueblo y Educación.

Junta de Superintendentes de la República de Cuba. (1926). Circulares y Planes de Estudio para las escuelas urbanas y rurales. Enseñanza Primaria Elemental. La Habana.

Martí, J. (1963). Obras Completas. Tomos: 5, 8 y 21. La Habana: Editorial Nacional de Cuba.

MES (2009). Programa Historia de Cuba. Universidad Central "Marta Abreu" de Las Villas. Santa Clara, Cuba.

MINED (1987). Concepción general de la asignatura Historia en el subsistema de la educación General Politécnica y Laboral. La Habana: Editorial Pueblo y Educación.
MINED (1996). Resolución Ministerial No. 60 de 1996. La Habana: Editorial Pueblo y Educación.

MINed (2001). Programas. De Primero a sexto grado; Programas. De séptimo a noveno grado; Programas. De décimo a duodécimo grado. La Habana: Editorial Pueblo y Educación.

MINED (2001). Orientaciones metodológicas. De primero a sexto grado. Tomos 1 y 2. De séptimo a noveno grado. Tomos 1 y 2. De décimo a duodécimo grado. Tomos 1 y 2. La Habana: Editorial Pueblo y Educación.

MINED (2001). Programa Director para las asignaturas priorizadas en la enseñanza primaria. La Habana: Editorial Pueblo y Educación. 


\section{DIÁLOGO DEL CONOCIMIENTO}

¿Para qué se enseña la historia? Es una pregunta cuya potencia se expresa en sus posibles respuestas, pues generalmente ponen sobre la mesa las intencionalidades de los proyectos políticos de las naciones, asociados en la mayoría de los casos, con la necesaria producción de sentimientos o emociones a favor de ideas, valores e imaginarios que se muestran consensuados y legitimados políticamente. ¿Qué hay más allá de los aparentes consensos?, ¿qué sucede con la diversidad existente en las profundidades sociales y culturales de las naciones? ¿qué otras historias se invisibilizan en los consensos?, ¿por qué son más visibles unas historias que otras?

Un efecto importante de los procesos de curricularización de los saberes escolares es la posibilidad de ganar un mayor control del Estado sobre lo que se enseña: ¿Qué se enseña?, ¿por qué se enseña? e incluso, ¿cómo se enseña? La historia es una especie de autobiografia que manifiesta lo que hemos venido siendo como nación. Sin importar el tipo de ideología o de tendencia política de los gobiernos de turno, permanece una vigilancia celosa sobre el pasado que se enseña, sobre la autobiografía de la nación que circula por las escuelas y colegios, incluso más, sobre las versiones de la historia que transitan por los medios y las redes.

Entonces, la escuela sigue siendo el "oasis" del pensamiento y del conocimiento, y el maestro, su artesano. Sigue siendo una institución que va a otro ritmo de lo que circula en lo social, que se detiene, experimenta, construye, y también, desaprende lo que social y culturalmente se ha legitimado; trasciende los tiempos y los espacios de las personas. Y el maestro, un sujeto que conduce el proceso, que tiene la inteligencia pedagógica para saber dónde detenerse, sobre qué experimentar, cómo construir y qué desaprender. La historia que se enseña podría subrayar el carácter histórico de lo que se conoce, de lo que pensamos y la manera cómo actuamos, de los espacios que transitamos y de lo que consumismos. La historia nos permite conocer la experiencia humana, pero tiene sentido con un horizonte de expectativa.

Mireya GonzÁlez 